\title{
Enzymic Basis For Leakiness of Auxotrophs for Phenylalanine in Pseudomonas aeruginosa
}

\author{
By ALAN BERRY, $\dagger$ RAJ K. BHATNAGAR AND ROY A. JENSEN* \\ Department of Microbiology and Cell Science, University of Florida, Gainesville, \\ Florida 32611, USA
}

(Received 10 February 1987; revised 9 July 1987)

\begin{abstract}
The dual enzymic routes for phenylalanine biosynthesis that exist in Pseudomonas aeruginosa complicate the isolation of phenylalanine auxotrophs. Mutants blocked in each of the various phenylalanine-pathway steps are essential for full appreciation of the physiological nature and gene-enzyme relationships of this biochemical system. A leaky phenylalanine-requiring mutant of $P$. aeruginosa (PAT1051) was found to lack the bifunctional P-protein (chorismate mutaseprephenate dehydratase), but retained the monofunctional isozyme species of chorismate mutase (chorismate mutase-F) as well as cyclohexadienyl dehydratase (components of the arogenate 'overflow' route to phenylalanine). This is the first mutant of $P$. aeruginosa shown to be deficient in any enzyme specific for phenylalanine synthesis. It is concluded that although the arogenate pathway has the demonstrated potential to overproduce phenylalanine, the substrate levels normally available to the arogenate pathway in the wild-type are inadequate to satisfy the full metabolic demand for phenylalanine.
\end{abstract}

\section{INTRODUCTION}

In Pseudomonas aeruginosa two enzymic routes exist for the biosynthesis of phenylalanine (Patel et al., 1977). This biochemical duality (depicted in Fig. 1), widely distributed in Gramnegative bacteria (Jensen, 1985), complicates the selection by conventional techniques of phenylalanine auxotrophs since the blocking of one enzymic route to phenylalanine is masked by the presence of the alternative route. Despite this biochemical basis for 'reluctant auxotrophy' (Patel et al., 1978), nutritional mutants requiring phenylalanine to sustain wild-type growth rates have been reported. Whitaker et al. $(1982 b)$ found that several leaky auxotrophs having a requirement for phenylalanine were deficient in one of five aminotransferases capable of functioning in phenylalanine and tyrosine synthesis. Waltho (1972) described six transduction groups of phenylalanine-responding mutants. Mutants belonging to five of these groups possessed chorismate mutase and prephenate dehydratase activities resembling those seen in the wild-type; they were thought to possess partial enzyme blocks in the common aromatic pathway, although specific enzyme deficiencies were not demonstrated. This assumption is indeed consistent with our finding that in $P$. aeruginosa, phenylalanine is the first of the aromatic amino acids to become rate-limiting to growth when flux through the common aromatic pathway is decreased. Thus, when moderately inhibitory concentrations of glyphosate, a specific inhibitor of 5-enolpyruvylshikimate-3-phosphate synthase (Steinrücken \& Amrhein, 1980), are administered to cultures of $P$. aeruginosa, phenylalanine alone restores the wild-type growth rate (A. Berry, unpublished data).

+ Present address: Department of Microbiology and Immunology, University of Illinois, Health Sciences Center at Chicago, Chicago, IL 60612, USA.

Abbreviation: DAHP, 3-deoxy-D-arabino-heptulosonate 7-phosphate. 


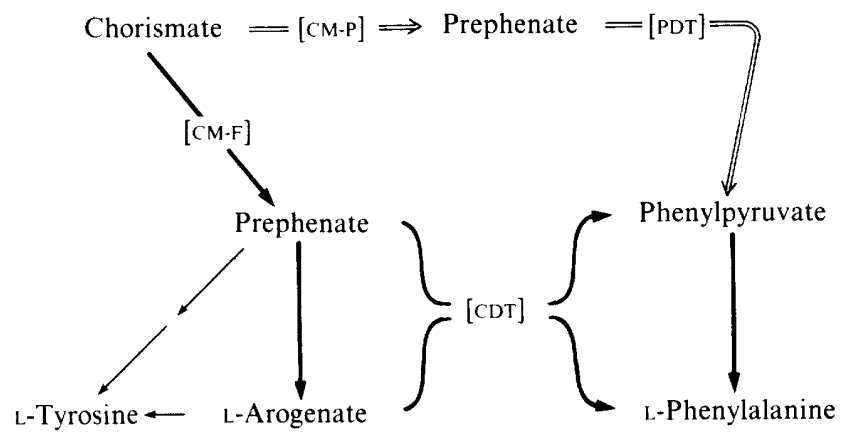

Fig. 1. Alternative enzymic routes for phenylalanine biosynthesis in $P$. aeruginosa. Enzyme abbreviations (in brackets) are: CM-F, chorismate mutase-F; CM-P, chorismate mutase-P; PDT, prephenate dehydratase; CDT, cyclohexadienyl dehydratase. CM-P and PDT (denoted by open arrows) are components of a single bifunctional enzyme (Calhoun et al., 1973a) denoted the P-protein (Cotton \& Gibson, 1965). CDT is capable of utilizing either $\mathrm{L}$-arogenate or prephenate as alternative cyclohexadienyl substrates (Patel et al., 1977). Both P-protein activities are feedback inhibited by Lphenylalanine; CM-P is additionally inhibited by prephenate, and PDT is activated by L-tyrosine (Calhoun et al., 1973a). CM-F is product inhibited by prephenate (A. Berry, unpublished results). CDT is not sensitive to feedback inhibition by L-phenylalanine, regardless of whether prephenate or Larogenate is utilized as substrate (Patel et al., 1977; Zamir et al., 1985). The conversions of phenylpyruvate to L-phenylalanine and prephenate to L-arogenate are transaminations apparently accomplished by a multiplicity of aminotransferases having overlapping specificities (Whitaker $e t$ al., $1982 b$ ). The 'overflow' pathway to L-phenylalanine is shown with bold arrows.

The P. aeruginosa transduction group PHE IV of Waltho (1972) was reported to consist of a mutant type lacking chorismate mutase. In view of the complexity of phenylalanine biosynthesis in $P$. aeruginosa, not known in 1972, a single mutation abolishing chorismate mutase is not likely. Since two chorismate mutase isozymes exist in $P$. aeruginosa, it seemed more probable that the quantitatively major isozyme, chorismate mutase-P (see Fig. 1), had been eliminated by mutation. This possibility seemed particularly likely since chorismate mutase-P channels chorismate toward phenylalanine (Calhoun et al., 1973a). In this report we show that a representative mutant of the PHE IV transduction group is not only deficient in chorismate mutase-P, but also lacks the second P-protein activity, prephenate dehydratase. This is the first mutant of $P$. aeruginosa known to be defective in any enzyme specific for phenylalanine synthesis, and the prospects for exploiting this mutant to probe the physiological nature of phenylalanine biosynthesis in $P$. aeruginosa are discussed.

\section{METHODS}

Strains and culture conditions. P. aeruginosa strains PAT2 (wild-type) and PAT1051 [a member of the PHE IV transduction group of phenylalanine-responding mutants (Waltho, 1972)] were obtained from B. W. Holloway (Department of Genetics, Monash University, Clayton, Victoria 3168, Australia). Cultures were grown at $37^{\circ} \mathrm{C}$ with shaking at 300 r.p.m. in the minimal-salts medium described by Fischer et al. (1986). Glucose $(0 \cdot 5 \%$, w/v) was used as the carbon source, and the medium was additionally supplemented with $50 \mu \mathrm{g}$ L-phenylalanine $\mathrm{ml}^{-1}$ wherever indicated.

Preparation and fractionation of crude extracts. Crude, desalted extracts were prepared as described by Byng $e t$ al. (1980) by sonication at $4{ }^{\circ} \mathrm{C}$ of cells harvested from 1 litre cultures. The extract buffer used was $50 \mathrm{~mm}$-EPPS/KOH, pH 7.9 (at $25^{\circ} \mathrm{C}$ ). Fractionation of crude extracts was done using $1.5 \times 20 \mathrm{~cm}$ columns of DEAE-cellulose (Whatman DE52) equilibrated with the buffer used for extract preparation (see above). Samples of crude extracts (72.9 mg protein and $84.8 \mathrm{mg}$ protein for PAT2 and PAT1051, respectively) were applied to separate columns, which were then washed with $100 \mathrm{ml}$ of equilibration buffer to remove unbound proteins. The bound proteins were then eluted with linear gradients of $\mathrm{KCl}(0-0.5 \mathrm{M})$, prepared in $300 \mathrm{ml}$ of equilibration buffer.

Enzyme assays. Chorismate mutase and prephenate dehydratase were assayed as described by Patel et al. (1977), while arogenate dehydratase was assayed using the HPLC method of Zamir et al. (1985). All reactions were done at $37^{\circ} \mathrm{C}$. Reaction mixture components are specified in the appropriate Figure and Table legends. Protein concentrations were estimated using the method of Bradford (1976). 
Materials. L-Arogenate was prepared according to Zamir et al. (1980, 1983). Barium prephenate was isolated from the culture supernatant of a tyrosine auxotroph of Salmonella typhimurium (Dayan \& Sprinson, 1970) and was converted to the potassium salt with 50 mM-potassium phosphate buffer $(\mathrm{pH} \mathrm{7.0)}$ before use. Chorismate was isolated from the medium of the triple auxotroph Klebsiella pneumoniae 62-1, and was purified as the free acid (Gibson, 1970). All other chemicals and Sephadex G-25 were obtained from Sigma. Protein assay reagent was obtained from Bio-Rad.

\section{RESULTS AND DISCUSSION}

\section{Comparison of enzyme properties of PAT1051 with the wild-type}

The wild-type parent strain, PAT2, is identical to P. aeruginosa PAO1 with respect to the enzymic arrangement and allosteric regulation of the aromatic amino acid pathway (A. Berry, unpublished observations). Thus, the published reports on aromatic-pathway enzymology in $P$. aeruginosa PAO1 (Calhoun et al., 1973a, b; Fiske et al., 1983; Jensen et al., 1973; Patel et al., 1977, 1978; Stenmark-Cox \& Jensen, 1975; Whitaker et al., 1982a,b) are also typical of the data obtained for $P$. aeruginosa strain PAT2.

PAT1051 possessed distinctly low activities for both chorismate mutase and prephenate dehydratase, compared to the wild-type parent PAT2 (Table 1). Since the bifunctional P-protein accounts for the major portion of chorismate mutase activity as well as for the majority of prephenate dehydratase in crude extracts, the data of Table 1 suggested a deficiency of the bifunctional P-protein (chorismate mutase-prephenate dehydratase; see Fig. 1). The residual chorismate mutase activity present in crude extracts of PAT1051 was that expected for the chorismate mutase-F isozyme, while the residual prephenate dehydratase activity could be due to cyclohexadienyl dehydratase. Cyclohexadienyl dehydratase, originally denoted arogenate dehydratase (Patel et al., 1977), recognizes both prephenate and L-arogenate as substrate (Jensen \& Fischer, 1986; Fischer \& Jensen, 1986). Partially purified cyclohexadienyl dehydratase exhibits a ratio of about five in comparison of activity with prephenate or L-arogenate as substrate under standard assay conditions, consistent with the ratio seen for these activities in mutant PAT1051.

The prephenate dehydratase component of the P-protein from wild-type PAT2 is inhibited more than $90 \%$ under standard assay conditions at phenylalanine concentrations exceeding $0.04 \mathrm{mM}$ (Fig. 2, inset). Fig. 2 gives the inhibition data obtained with a crude extract of PAT2, showing inhibition to level off at $55-60 \%$. This result is consistent with expectations for a crude extract containing a mixture of feedback-resistant enzyme molecules (cyclohexadienyl dehydratase) and feedback-sensitive enzyme molecules (prephenate dehydratase). If the

Table 1. Altered enzyme activities in strain PAT1051

Crude extracts were prepared in $50 \mathrm{~mm}$-EPPS buffer $\left(\mathrm{pH} 7.9\right.$ at $\left.25^{\circ} \mathrm{C}\right)$ as described in the text. All reaction mixtures contained 50 mM-EPPS buffer $\left(\mathrm{pH} 7.9\right.$ at $25^{\circ} \mathrm{C}$ ), substrate (at the concentration specified below), and crude-extract protein in a final volume of $200 \mu \mathrm{l}$. Chorismate mutase, prephenate dehydratase and cyclohexadienyl dehydratase were assayed using final substrate concentrations of $1.0 \mathrm{~mm}$-chorismate, $1.0 \mathrm{~mm}$-prephenate, and $1.1 \mathrm{mM}-\mathrm{L}$-arogenate, respectively.

Specific activity

[nmol product $\min ^{-1}(\mathrm{mg} \text { protein })^{-1}$ ]

\begin{tabular}{|c|c|c|c|c|}
\hline \multirow[b]{3}{*}{ Strain } & \multicolumn{4}{|c|}{$\begin{array}{c}\text { Specific activity } \\
{\left[\text { nmol product } \min ^{-1}(\text { mg protein })^{-1}\right]}\end{array}$} \\
\hline & \multirow[b]{2}{*}{$\begin{array}{l}\text { Arogenate } \\
\text { dehydratase }\end{array}$} & \multirow[b]{2}{*}{$\begin{array}{c}\text { Chorismate } \\
\text { mutase }\end{array}$} & \multicolumn{2}{|c|}{$\begin{array}{l}\text { Prephenate } \\
\text { dehydratase* }\end{array}$} \\
\hline & & & -Phe & $+\mathrm{Phe}$ \\
\hline PAT2 & $3 \cdot 6$ & $31 \cdot 0$ & $66 \cdot 0$ & $22 \cdot 0$ \\
\hline PAT105I & $2 \cdot 8$ & $4 \cdot 2$ & $15 \cdot 3$ & 13.6 \\
\hline
\end{tabular}

* Determined in the absence $(-\mathrm{Phe})$ or presence $(+\mathrm{Phe})$ of $100 \mu \mathrm{M}$-L-phenylalanine. 


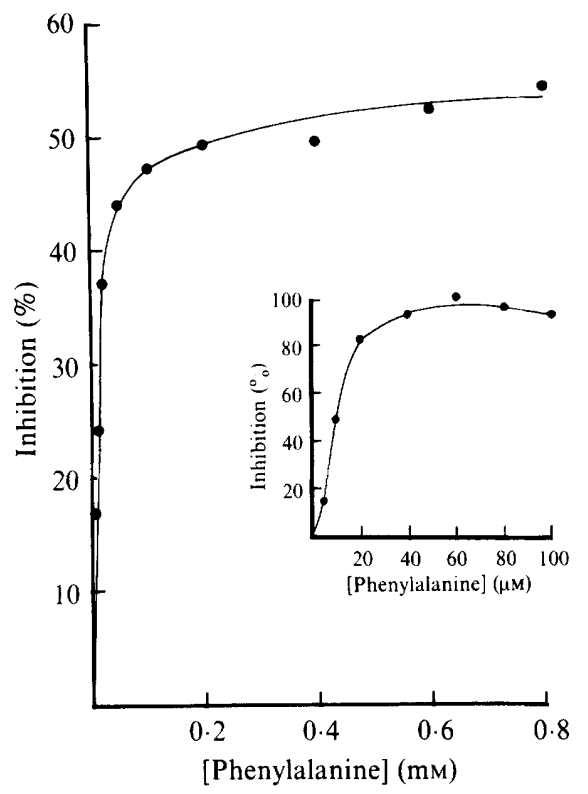

Fig. 2. Phenylalanine inhibition of prephenate dehydratase in a crude extract of wild-type $P$. aeruginosa PAT2. Crude extract was prepared in 50 mM-EPPS buffer (pH 7.9 at $25^{\circ} \mathrm{C}$ ) as described in the text. Reaction mixtures contained $1.0 \mathrm{~mm}$-prephenate, the aforementioned EPPS buffer, enzyme and the indicated concentration of L-phenylalanine in a final volume of $200 \mu \mathrm{l}$. Inset: Phenylalanine inhibition of partially purified prephenate dehydratase (P-protein) from PAT2 obtained following fractionation of a crude extract by DEAE-cellulose chromatography (see Fig. 3). Reaction mixtures were the same as described above for crude extract.

prephenate dehydratase activity present in PAT1051 is exclusively attributed to cyclohexadienyl dehydratase, then one would expect insensitivity to inhibition by phenylalanine because only the P-protein dehydratase of $P$. aeruginosa is sensitive to inhibition by phenylalanine (Calhoun et al., 1973a; Patel et al., 1977; Zamir et al., 1985). The data given in Table 1 show that the latter expectations were borne out, although prephenate dehydratase activity in PAT1051 was inhibited about $11 \%$ by phenylalanine. The error in the assay is no greater than $5 \%$, and three different experiments gave a mean value of about $15 \%$ for inhibition of total prephenate dehydratase activity in PAT1051. Inhibition by antibody raised against purified P-protein from Acinetobacter calcoaceticus (courtesy of S. Ahmad) gave results consistent with as much as $14 \%$ of the prephenate dehydratase activity of PAT1051 being due to P-protein dehydratase. If so, PAT 1051 may express as much as $5 \%$ of the normal P-protein dehydratase. Mutant PAT1051 is very stable and has never been observed to revert, even though selection for revertants during slow growth on minimal medium would be very strong. Perhaps a multi-site deletion has abolished the promoter and/or the promoter-proximal chorismate mutase portion of the Pprotein gene, leaving a weak internal promoter that can account for minor expression of a feedback-sensitive prephenate dehydratase. Thus far, all attempts to find any dehydratase activity separable from cyclohexadienyl dehydratase in PAT1051 have yielded negative results (see next section).

The specific activity of cyclohexadienyl dehydratase was consistently lower in PAT1051 than in PAT2. In three different experiments PAT 1051 possessed about $70 \%$ of the activity found in PAT2. (The error in the assay of cyclohexadienyl dehydratase with L-arogenate was no greater than $7 \%$.) This phenomenon was not related to faster growth of PAT2 than of PAT1051 in minimal medium since phenylalanine-grown cultures gave similar results. The data also indicated that phenylalanine excess does not repress and phenylalanine limitation (PAT1051 grown without phenylalanine) does not derepress cyclohexadienyl dehydratase. 


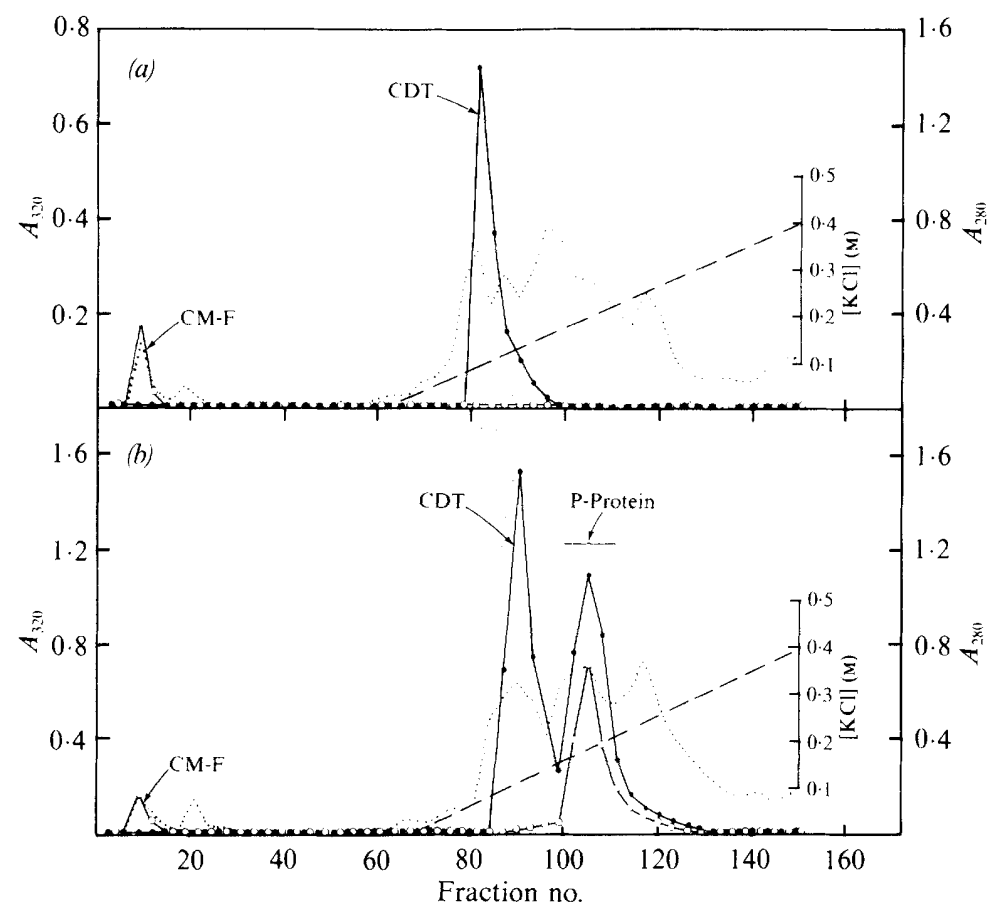

Fig. 3. Elution profiles of chorismate mutase isoenzymes, prephenate dehydratase and cyclohexadienyl dehydratase (CDT) from $P$. aeruginosa PAT1051 (a) and PAT2 (b) following fractionation of crude extracts by DEAE-cellulose chromatography as described in the text. Prephenate dehydratase activity $(O)$ and chorismate mutase activity $(O)$ are expressed as phenylpyruvate absorbance at $320 \mathrm{~nm}$. For convenience, CDT activity peaks were initially located using prephenate as substrate. The identities of these peaks were then confirmed by assaying peak fractions using L-arogenate as substrate. The specific activities for the CDT peak fractions (using L-arogenate as substrate) were 44 and $25 \mathrm{nmol}$ product formed $\min ^{-1}$ (mg protein) ${ }^{-1}$ for PAT2 and PAT1051, respectively. The prephenate dehydratase (P-protein) from PAT2 was totally unreactive with $L$-arogenate as substrate. All reaction mixtures contained $50 \mathrm{mM}$-EPPS buffer $\left(\mathrm{pH} 7.9\right.$ at $25^{\circ} \mathrm{C}$ ), a $1.0 \mathrm{~mm}$ final concentration of the assay substrate and eluate protein in a final volume of $200 \mu \mathrm{l}$..., Distribution of protein measured as absorbance at $280 \mathrm{~nm} ;---, \mathrm{KCl}$ gradient.

Chromatographic resolution of enzymes of phenylalanine biosynthesis from PAT2 and PAT1051

The lack of the P-protein in PAT1051 was confirmed by fractionating crude extracts by DEAE-cellulose chromatography. Fig. 3 shows a comparison of the elution profiles for chorismate mutase, prephenate dehydratase and cyclohexadienyl dehydratase from PAT2 $(b)$ and PAT1051 (a). Total chorismate mutase activity from PAT2 eluted as two distinct peaks, a minor peak (isozyme chorismate mutase-F) and a major peak (isozyme chorismate mutase-P). Chorismate mutase-P co-eluted exactly with prephenate dehydratase, consistent with their joint physical association as a bifunctional P-protein (Patel et al., 1977). Cyclohexadienyl dehydratase from PAT2 eluted earlier than the P-protein in the $\mathrm{KCl}$ gradient.

Only one peak of chorismate mutase activity was recovered from PAT1051 (Fig. $3 a$ ), which corresponded to the chorismate mutase-F isozyme. Cyclohexadienyl dehydratase activity was detected in the gradient fractions, as was expected from the presence of this activity in crude extract (see Table 1). Both activities of the P-protein were absent in PAT1051. Additional enzymic deficiencies were not detected in PAT1051 (data not shown). Thus, this strain resembled the wild-type with respect to the presence of two regulatory isozymes of 3-deoxy-Darabino-heptulosonate-7-phosphate (DAHP) synthase, dehydroshikimate reductase, 5-enolpyruvylshikimate-3-phosphate synthase, prephenate dehydrogenase, arogenate dehydrogenase 
and five separate aromatic aminotransferase activities (Calhoun et al., 1973a, b; Fischer et al., 1986; Fiske et al., 1983; Jensen et al., 1973; Patel et al., 1977, 1978; Stenmark-Cox \& Jensen, 1975; Whitaker et al., 1982a, b).

The prephenate dehydratase component of the $P$. aeruginosa P-protein is activated by Ltyrosine (Calhoun et al., 1973a). In Xanthomonas campestris L-tyrosine not only activates the prephenate dehydratase of the P-protein, but is also required for stability (Whitaker et al., 1985). This and the observation that L-tyrosine stimulates the growth rate of $P$. aeruginos $a$ PAT1051, suggested that PAT1051 might possess a labile P-protein that could be protected by L-tyrosine. However, an elution profile identical to that shown in Fig. 3(a) was obtained when a crude extract of PAT1051 was prepared and fractionated in the presence of $1.0 \mathrm{mM}$-L-tyrosine (data not shown).

\section{Biochemical and physiologial implications}

We have shown that $P$. aeruginosa PAT1051 is deficient in the bifunctional P-protein (chorismate mutase-prephenate dehydratase), but rotains both the minor isozyme of chorismate mutase (chorismate mutase-F) and cyclohexadienyl dehydratase. The joint elimination of both chorismate mutase-P and prephenate dehydratase as the consequence of a single mutation supports the contention that both catalytic activities are the property of a single protein. This bifunctional protein appears to be distributed throughout two of three major superfamilies of the Gram-negative bacteria (Ahmad \& Jensen, 1986).

PAT1051 marks a starting point for strategies of selection for other mutant deficiencies in the post-prephenate portion of the aromatic pathway (see Fig. 1). For example, elimination of the Pprotein in PAT1051 leaves only one route to phenylalanine intact (via cyclohexadienyl dehydratase). It should now be possible to isolate tight phenylalanine auxotrophs as single-step mutants by eliminating cyclohexadienyl dehydratase. It may then be possible to study mutants that are deficient only in cyclohexadienyl dehydratase following restoration of the P-protein gene. Secondly, the absence of chorismate mutase-P in PAT1051 leaves only chorismate mutase$F$ to provide all of the prephenate molecules needed for phenylalanine and tyrosine synthesis. Hence, a mutation that eliminates chorismate mutase-F in the genetic background of PAT1051 should yield a phenotype of auxotrophy for both phenylalanine and tyrosine. Nutritional and physiological studies of mutants lacking each of the pathway enzymes will provide insight into the significance of the dual-pathway arrangement which has proven to be widely distributed among Gram-negative bacteria (Jensen, 1985).

It has been shown that when DAHP synthase-tyr, the tyrosine-sensitive major isozyme of DAHP synthase in $P$. aeruginosa (Whitaker et al., 1982a), loses sensitivity to allosteric control by mutation, phenylalanine is overproduced owing to the absence of end-product control for the 'overflow' pathway (consisting of chorismate mutase- $F$ and cyclohexadienyl dehydratase) (Fiske et al., 1983). Presumably, wild-type cells do not excrete phenylalanine because the overflow pathway requires elevated levels of phenylalanine-pathway precursors, a circumstance prevented by the controlling influence of tyrosine upon DAHP synthase-tyr. Our present results show that in the absence of the P-protein route through phenylpyruvate, the overflow pathway through L-arogenate and/or phenylpyruvate is not even adequate to provide sufficient phenylalanine to sustain the normal wild-type rate of growth.

These studies were supported by grant AM-38309 from the US Public Health Service.

This paper is Florida Agricultural Station Journal Series No. 8433.

\section{REFERENCES}

Ahmad, S. \& Jensen, R. A. (1986). The evolutionary history of two bifunctional proteins that emerged in the purple bacteria. Trends in Biochemical Science 11, 108-112.

BRADFORD, M. M. (1976). A rapid and sensitive method for the quantitation of microgram quantities of protein utilizing the principle of protein-dye binding. Analytical Biochemistry 72, 248-254.
Byng, G. S., Whitaker, R. J., Gherna, R. L. \& JENSEN, R. A. (1980). Variable enzymological patterning in tyrosine biosynthesis as a means of determining natural relatedness among the Pseudomonadaceae. Journal of Bacteriology 144, 247-257.

Calhoun, D. H., Pierson, D. L. \& Jensen, R. A. (1973a). Channel-shuttle mechanism for the regulation of phenylalanine and tyrosine synthesis at a 
metabolic branchpoint in Pseudomonas aeruginosa. Journal of Bacteriology 113, 241-251.

Calmoun, D. H., Pierson, D. L. \& Jensen, R. A. $(1973 b)$. The regulation of tryptophan biosynthesis in Pseudomonas aeruginosa. Molecular and General Genetics 121, 117-132.

Cotron, R. G. H. \& Gibson, F. (1965). The biosynthesis of phenylalanine and tyrosine: enzymes converting chorismic acid into prephenic acid and their relationships to prephenate dehydratase and prephenate dehydrogenase. Biochimica et biophysica acta 100, 76-88.

DAYAN, J. \& SPrinson, D. B. (1970). Preparation of prephenic acid. Methods in Enzymology 17A, 559561.

FISCHER, R. \& JENSEN, R. A. (1986). Arogenate dehydratase. Methods in Enzymology 142, 495-502.

Fischer, R. S., Berry, A., Gaines, C. G. \& Jensen, R. A. (1986). Comparative action of glyphosate as a trigger of energy drain in eubacteria. Journal of Bacteriology 168, 1147-1154.

Fiske, M. J., WhitakeR, R. J. \& JenSEN, R. A. (1983). Hidden overflow pathway to L-phenylalanine in Pseudomonas aeruginosa. Journal of Bacteriology 154, 623-631.

Gibson, F. (1970). Preparation of chorismic acid. Methods in Enzymology 17A, 362-364.

JENSEN, R. A. (1985). Biochemical pathways can be traced backward through evolutionary time. $\mathrm{Molecu}$ lar Biology and Evolution 2, 92-108.

Jensen, R. A., Calhoun, D. H. \& Stenmark, S. L. (1973). Allosteric inhibition of 3-deoxy-D-arabinoheptulosonate 7-phosphate synthetase by tyrosine, tryptophan, and phenylpyruvate in Pseudomonas aeruginosa. Biochimica et biophysica acta 293, 256268.

Jensen, R. A. \& Fischer, R. (1986). The postprephenate biochemical pathways to phenylalanine and tyrosine: an overview. Methods in Enzymology 142, 472-478.

Patel, N., Pierson, D. L. \& Jensen, R. A. (1977). Dual enzymatic routes to L-tyrosine and L-phenylalanine via pretyrosine in Pseudomonas aeruginosa. Journal of Biological Chemistry 252, 5839-5846.

Patel, N., Stenmark-Cox, S. L. \& Jensen, R. A. (1978). Enzymological basis of reluctant auxotrophy for phenylalanine and tyrosine in Pseudomonas aeruginosa. Journal of Biological Chemistry 253, 29722978.

Steinrücken, H. C. \& AmRHein, N. (1980). The herbicide glyphosate is a potent inhibitor of 5 enolpyruvylshikimic acid-3-phosphate synthase. Biochemical and Biophysical Research Communications 94, 1207-1212.

Stenmark-Cox, S. \& Jensen, R. A. (1975). Prephenate dehydrogenase from Pseudomonas aeruginosa is a regulated component of the channel-shuttle mechanism controlling tyrosine-phenylalanine synthesis. Archives of Biochemistry and Biophysics 167, 540-546.

Waltho, J. A. (1972). Genetic analysis of phenylalanine-responding mutants of Pseudomonas aeruginosa. Journal of Bacteriology 112, 1070-1075.

Whitaker, R. J., Fiske, M. J. \& Jensen, R. A. (1982a). Pseudomonas aeruginosa possesses two novel regulatory isozymes of 3-deoxy-D-arabino-heptulosonate 7-phosphate synthase. Journal of Biological Chemistry 257, 12789-12794.

Whitaker, R. J., Gaines, C. G. \& Jensen, R. A. (1982b). A multispecific quintet of aromatic aminotransferases that overlap different biochemical pathways in Pseudomonas aeruginosa. Journal of Biological Chemistry 257, 13550-13556.

WhitAKER, R. J., BERRY, A., BYNG, G. S., Fiske, M. J. \& JENSEN, R. A. (1985). Clues from Xanthomonas campestris about the evolution of aromatic biosynthesis and its regulation. Journal of Molecular Evolution 21, 139-149.

ZamiR, L. O., Jensen, R. A., Arison, B. H., Douglas, A. W., Albers-Schonberg, G. \& BOWEN, J. R. (1980). Structure of arogenate (pretyrosine), an amino acid and intermediate of aromatic biosynthesis. Journal of the American Chemical Society 102 , 4499-4504.

ZAmiR, L. O., Jung, E. \& Jensen, R. A. (1983). Coaccumulation of prephenate, $L$-arogenate, and spiroarogenate in a mutant of Neurospora. Journal of Biological Chemistry 258, 6492-6496.

ZAMIR, L. O., TIBERIo, R., Fiske, M., Berry, A. \& JENSEN, R. A. (1985). Enzymatic and non-enzymatic dehydration reactions of L-arogenate. Biochemistry 24, 1607-1612. 